LAW RENCE LIVERMORE N A T IO N A L LABORATORY
Incorporating the Bowen Survivability Curves into Blast Analysis

L. D. McMichael

January 31, 2011 
This document was prepared as an account of work sponsored by an agency of the United States government. Neither the United States government nor Lawrence Livermore National Security, LLC, nor any of their employees makes any warranty, expressed or implied, or assumes any legal liability or responsibility for the accuracy, completeness, or usefulness of any information, apparatus, product, or process disclosed, or represents that its use would not infringe privately owned rights. Reference herein to any specific commercial product, process, or service by trade name, trademark, manufacturer, or otherwise does not necessarily constitute or imply its endorsement, recommendation, or favoring by the United States government or Lawrence Livermore National Security, LLC. The views and opinions of authors expressed herein do not necessarily state or reflect those of the United States government or Lawrence Livermore National Security, LLC, and shall not be used for advertising or product endorsement purposes.

This work performed under the auspices of the U.S. Department of Energy by Lawrence Livermore National Laboratory under Contract DE-AC52-07NA27344. 


\title{
Incorporating the Bowen Survivability Curves into Blast Analysis
}

\author{
Larry McMichael \\ Lawrence Livermore National Laboratory \\ January 27, 2011
}

\section{Bowen Relationship for Surviving a Blast Wave}

The survival probability for exposure to a sharp-rising blast wave is expressed by Figure 3 in Bowen's 1968 report, Estimate of Man's Tolerance to the Direct Effects of Air Blast. The relationship is based on mortality data for a mixture of mammalian species that were exposed to blast overpressures near a reflecting surface. The derived relationship is expressed in terms of a scaled peak reflected overpressure, $p_{r}$, and scaled duration, $T$. The scaled peak reflected overpressure is adjusted for a specie's tolerance to blast overpressures $\left(P_{s w}\right)$ and the ambient air pressure, $p_{a t m}$. The scaled duration is adjusted for body mass, $m$, and the ambient air pressure. The curves are therefore meant to be adapted for variations in altitude $\left(p_{a t m}\right)$, blast tolerance $\left(P_{s w}\right)$, and body mass (adult, child). The primary curve is based upon $50 \%$ mortality and probit analysis is used to establish the other survivability curves. The probit values, $Z$, associated with some survivability levels are given below.

Table 1: Probit values associated with some survivability levels.

\begin{tabular}{|c|c|}
\hline Probability of Survival & Probit Value, $Z$ \\
\hline \hline $99 \%$ & 7.33 \\
\hline $90 \%$ & 6.28 \\
\hline $75 \%$ & 5.67 \\
\hline $50 \%$ & 5.00 \\
\hline $25 \%$ & 4.33 \\
\hline $10 \%$ & 3.72 \\
\hline $1 \%$ & 2.67 \\
\hline
\end{tabular}

The relationship determined by Bowen to describe the mortality data is given by:

$$
p_{r}\left(\frac{61.5}{P_{s w}}\right)\left(\frac{14.7}{p_{a t m}}\right)=61.5\left(1+6.76 T^{-1.064}\right) e^{0.1788(5-Z)}
$$

Where the pressures $p_{r}$ and $p_{a t m}$ are in psi, and the scaled duration $T$ is in msec. The scaled duration is given by:

$$
T=t_{d u r}\left(\frac{70}{m}\right)^{\frac{1}{3}}\left(\frac{14.7}{p_{a t m}}\right)^{\frac{1}{2}}
$$

where the mass $m$ is in $\mathrm{kg}$ and $t_{d u r}$ is the positive phase duration of the overpressure at the reflecting surface. Bowen estimated man's blast tolerance as $P_{s w}=61.5$, so if the 
relationships in Equation 1 and 2 are simplified for a $70 \mathrm{~kg}$ person and a blast near sea level (i.e., $p_{a t m}=14.7 \mathrm{psi}$ ), then $T=t_{d u r}$ and the expression for $p_{r}$ becomes:

$$
p_{r}=61.5\left(1+6.76 t_{d u r}^{-1.064}\right) e^{0.1788(5-Z)}
$$

\section{Effect of Body Orientation}

Survivability is influenced by several factors, including a person's body position relative to the blast wave and the presence or absence of a reflecting surface. To extrapolate the mortality data represented in Figure 3 of Bowen's report to other scenarios, Bowen postulated the conditions needed for equivalent biological damage to relate the free field (incident) pressures experienced for a particular body orientation to the measured peak reflected pressures in the experiments. For the orientations considered in Bowen's report, the survivability curves are generated for a $70 \mathrm{~kg}$ person near sea level, which is consistent with Equation (3) above.

\section{Next to Reflecting Surface}

An example of this extrapolation is the generation of the survivability curves in Figure 7 of Bowen's report for a person standing next to a reflecting surface. In essence, this case simply transforms the survivability relationship into terms of incident pressure, $p_{i}$, rather than reflected pressure, $p_{r}$. Although Bowen does not explicitly state in the report what relationship is used to convert the reflected pressure to incident pressure, the curves can be replicated using the expression from Glasstone's The Effects of Nuclear Weapons relating reflected pressure to incident pressure for an ideal gas with a constant specific heat ratio, $\gamma$ :

$$
p_{r}=2 p_{i}\left(\frac{7 p_{a t m}+4 p_{i}}{7 p_{a t m}+p_{i}}\right)
$$

Solving this relationship for $p_{i}$ leads to:

$$
p_{i}=\frac{\left(p_{r}-14 p_{a t m}\right)+\sqrt{\left(14 p_{a t m}-p_{r}\right)^{2}+224 p_{a t m} p_{r}}}{16}
$$

where $p_{r}$ is determined from Equation (3).

\section{Prone Position}

For a prone position, where the long axis of the body is parallel to the blast wave propagation, Bowen postulated that equivalent biological damage would occur if the incident pressure was equal to the reflected pressure in Figure 3. The expression for the incident pressure is obtained by simply substituting $p_{i}$ for $p_{r}$ in Equation (3):

$$
p_{i}=61.5\left(1+6.76 t_{d u r}^{-1.064}\right) e^{0.1788(5-Z)}
$$




\section{Standing Position}

The third body orientation considered by Bowen was standing, i.e. the long axis of the body is perpendicular to the blast wave propagation. This body orientation also corresponds to a prone position where the blast wave approaches from the person's side. For this body position, Bowen postulated that the incident pressure plus dynamic pressure must equal the measured reflected pressure for equivalent biological damage to occur. Again, Bowen does not specify the relationship used to relate dynamic pressure, $q$, to the incident pressure, but the curves in Figure 6 of Bowen's report can be replicated using an expression from Glasstone. The peak dynamic pressure is determined from the density $\rho$ and particle velocity $u$ through the Rankine-Hugoniot relationships and the assumption of a constant $\gamma$ ideal gas:

$$
q=\frac{5}{2}\left(\frac{p_{i}^{2}}{7 p_{a t m}+p_{i}}\right)
$$

Setting $p_{i}+q=p_{r}$ and substituting the above equation for $q$ leads to:

$$
p_{i}=\frac{\left(p_{r}-7 p_{a t m}\right)+\sqrt{\left(7 p_{a t m}-p_{r}\right)^{2}+98 p_{a t m} p_{r}}}{7}
$$

where $p_{r}$ is determined from Equation (3).

\section{Survivability in a Tunnel}

The overpressures experienced from a blast in an enclosed space, such as a tunnel, result from a complex series of pressure wave interactions and reflections. The overpressure time history will consequently have a series of peak pressures and will typically possess a longer positive phase duration than free field exposure. There is some biological evidence that the subsequent pressure peaks are less biologically damaging than the initial peak pressure, since the body is already exposed to a higher "ambient" pressure, as though the blast had occurred at a higher atmospheric pressure. Therefore, in the same nature as the body orientation, the complex overpressure time history experienced within the tunnel needs to be related to the single, sharp rising pressure pulse underlying the experimental data expressed by the Bowen curves in order to determine equivalent biological damage. Some partial impulse methods have been proposed that use the peak overpressure in combination with the impulse over the first two to three milliseconds of the blast exposure to correlate biological damage from the complex wave to a single wave. However, the experimental data used to derive the partial impulse methods tends to be for small enclosed spaces, such as armored personnel carriers or other fortifications with relatively small openings to vent the explosive overpressures. The vent opening for a tunnel is effectively its cross-sectional area, so pressure relief in the tunnel will occur much faster than for a small enclosed space with limited venting. The faster rate of pressure relief in tunnels would likely mitigate the increased resistance observed for complex blast waves in an enclosed space.

The intended purpose for this work is the prediction of the extent of injuries associated with an explosive detonation in order to assist transit system stakeholders in evaluating mitigation options and response planning. Within this context, basing equivalent biological damage on the total positive phase duration experienced in the tunnel is a conservative 
assumption and will be used for this work. Since most people will not be directly next to a reflecting surface, the assumed body orientation is a standing position, which is the most vulnerable free-field position. The baseline assumptions of $70 \mathrm{~kg}$ body mass and standard atmospheric pressure will also be used. Under these assumptions, the applicable survivability equations are (3) and (8) in combination with the probit values listed in Table 1.

\section{Incorporation into Hydrocode Analysis}

In a typical hydrocode analysis, an artificial viscosity term is used to smear the sharp pressure rise associated with a shock front across multiple elements in order to promote numerical stability in the solution. The artificial viscosity therefore acts, in essence, as part of the total pressure in the simulation. The total pressure in the simulation is then: $p_{t o t}=p+q_{a v}$, where $p$ is the zonal pressure and $q_{a v}$ is the artificial viscosity. The overpressure in the simulation can then be expressed as:

$$
p_{o p}=p_{t o t}-p_{a t m}=p+q_{a v}-p_{a t m}
$$

The overpressure $p_{o p}$ will include contributions from various reflections within the tunnel, but it essentially represents the incident overpressure for that zone. The maximum overpressure $p_{o p}^{\max }$ in a zone can be tracked over the simulation to develop a spatial and temporal distribution of maximum overpressure over the domain. Similarly, the amount of time that the overpressure in a zone is greater than $p_{a t m}$ can be tracked to establish each zone's positive phase duration, $t_{z}$. The zone's positive phase duration $t_{z}$ is then used in Equation (3) along with a probit value from Table 1 to determine the maximum reflected pressure $p_{r}$ associated with the probit's survivability level for that duration of exposure. The reflected pressure is used in Equation (8) to determine the maximum incident pressure $p_{i}$ associated with that survivability level (probit value) and duration. If $p_{o p}^{\max }$ is greater than $p_{i}$, then that zone exceeds the threshold for that survivability level.

\section{Example Survivability Calculation}

As an example for determining the appropriate survivability level, consider a hydrocode simulation that returns a peak incident overpressure of $p_{o p}^{\max }=130$ psi and positive phase duration of $t_{z}=1.2 \mathrm{msec}$ for a particular point. For this example, consider only the $1 \%$, $50 \%$ and $99 \%$ survivability levels.

1. Evaluate survivability relative to the $1 \%$ survivability curve

- Determine the $p_{r}$ for $1 \%$ survivability from Equation (3) by setting $t_{d u r}=t_{z}=1.2$ msec and $Z=2.67: p_{r}=612.7$ psi

- Determine the $p_{i}$ for $1 \%$ survivability from Equation (8) by setting $p_{r}=612.7$ psi and $p_{\text {atm }}=14.7$ psi: $p_{i}=225.5$ psi

- $p_{o p}^{\max }<p_{i}$ for $1 \%$ survivability, so survivability is better than $1 \%$

2. Evaluate survivability relative to the $50 \%$ survivability curve 
- Determine the $p_{r}$ for $50 \%$ survivability from Equation (3) by setting $t_{d u r}=t_{z}=1.2$ msec and $Z=5.0: p_{r}=403.9 \mathrm{psi}$

- Determine the $p_{i}$ for $50 \%$ survivability from Equation (8) by setting $p_{r}=403.9$ psi and $p_{\text {atm }}=14.7$ psi: $p_{i}=160.2$ psi

- $p_{o p}^{\max }<p_{i}$ for $50 \%$ survivability, so survivability is better than $50 \%$

3. Evaluate survivability relative to the $99 \%$ survivability curve

- Determine the $p_{r}$ for $99 \%$ survivability from Equation (3) by setting $t_{d u r}=t_{z}=1.2$ msec and $Z=7.33: p_{r}=266.3$ psi

- Determine the $p_{i}$ for $99 \%$ survivability from Equation (8) by setting $p_{r}=266.3$ psi and $p_{a t m}=14.7$ psi: $p_{i}=114.8$ psi

- $p_{o p}^{\max }>p_{i}$ for $99 \%$ survivability, so the survivability level is between $99 \%$ and $50 \%$

This methodology determines the expected survivability relative to the survivability curves evaluated, so the range in the survivability level is dependent upon how many curves are evaluated. In the above example, only three survivability curves are used, so the survivability range is rather large, $50 \%$ to $99 \%$. Additional probit values are available beyond those listed in Table 1, so the survivability range could be determined to the nearest percent. However, given the uncertainty embedded in Bowen's survivability relationship (i.e., extrapolating the test data to humans)and the assumed conditions for equivalent biological damage, most applications can utilize five to ten survivability curves for a reasonable resolution of the survivability range.

\section{Example Simulation}

A blast in a simple, generic tunnel, refer to Figure 1, was simulated to demonstrate the above methodology. For each zone, the maximum overpressure during the simulation was tracked, and the positive phase duration was calculated. The spatial distribution of those quantities is shown in Figure 2. Based upon those values and the Bowen relationships described above, the human survivability contours were estimated over the problem domain, refer to Figure 3. In Figure 3, red indicates that there is a 1-to-10\% chance of survival, while orange indicates 10-to-25\% survivability. The simulations reflected in Figure 3 are for equal charge weights, but different geometries and detonation assumptions: a spherical charge with (instantaneous) volume burn and a cylindrical charge with a detonator (programmed burn) on the end furthest from the end wall, i.e. the blast is focused towards the back of the tunnel. The spatial variations in the projected survivability contours outside the tunnel provide an indication of the influence that charge geometry and detonation conditions can have on survivability estimates well away from the threat. 


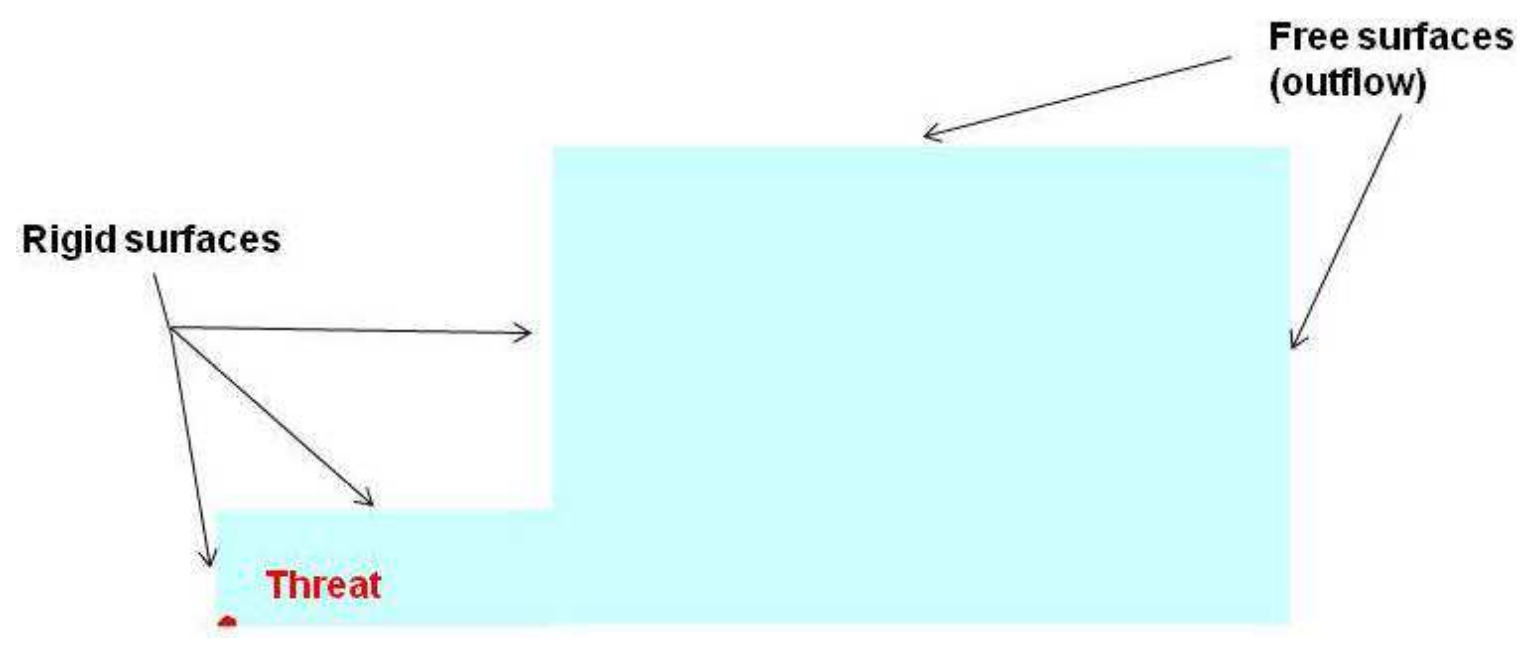

Figure 1: Generic tunnel geometry and boundary conditions.

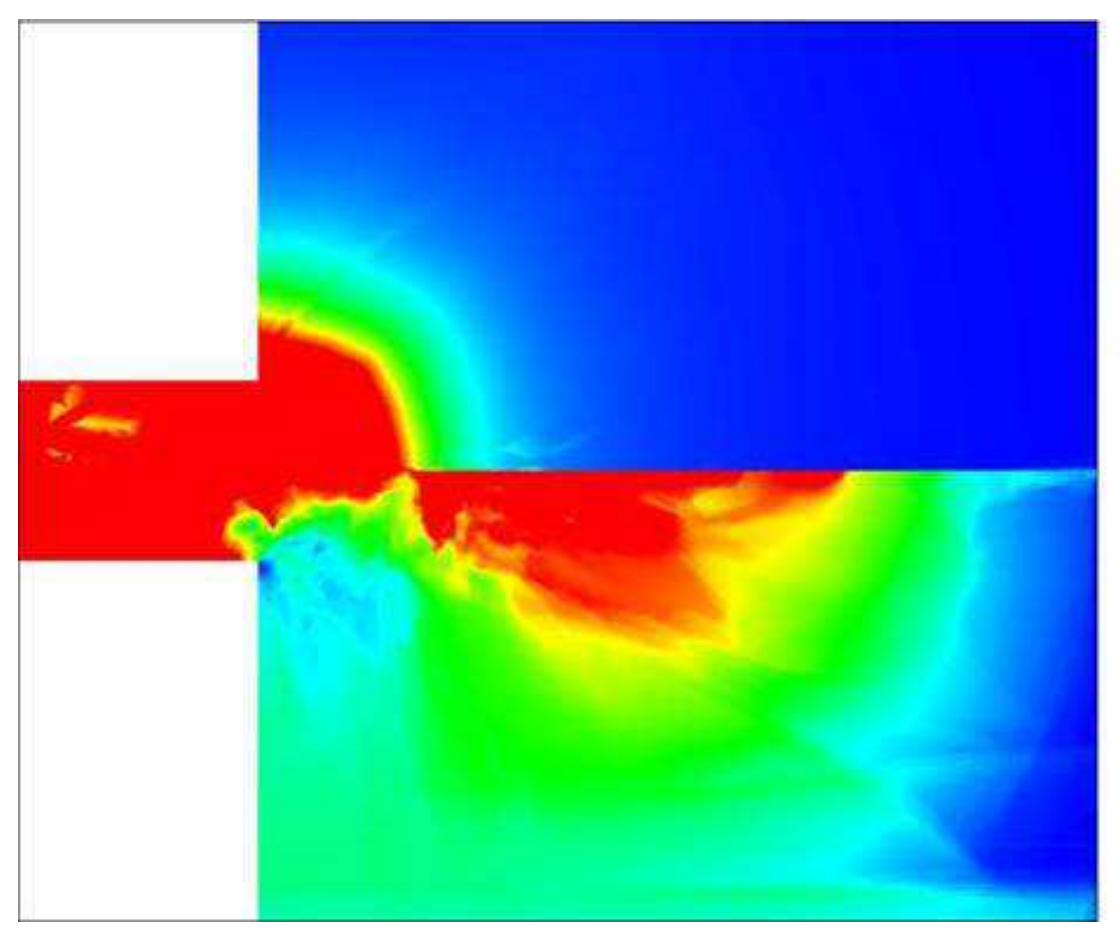

Maximum

Overpressure

\section{Duration}

Figure 2: Maximum overpressure and overpressure duration from the simulation. 

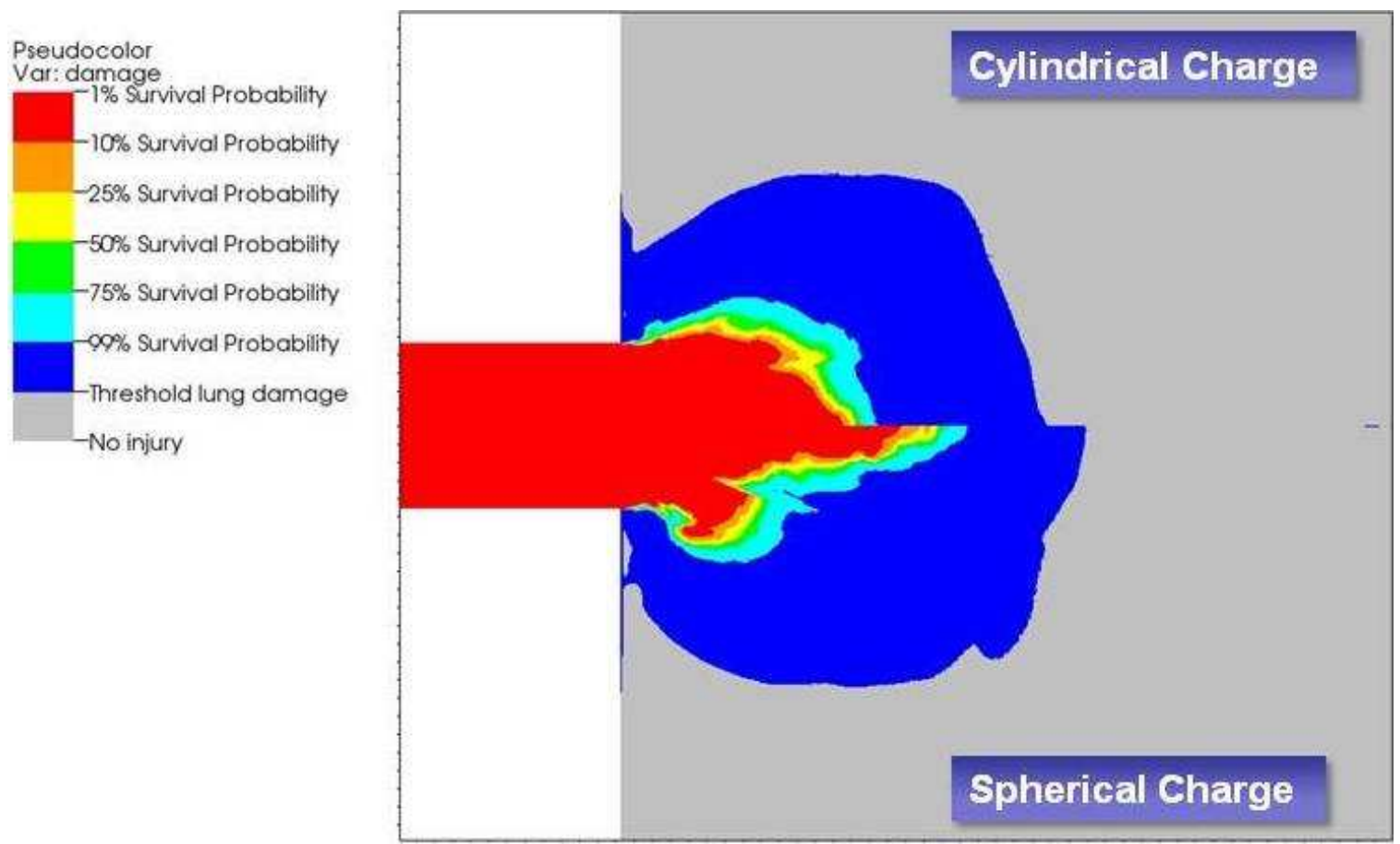

Figure 3: Survivability contours determined for different idealized threats. 